Check for updates

Cite this: RSC Adv., 2021, 11, 24607

\title{
Optimized iminium-catalysed 1,4-reductions inside the resorcinarene capsule: achieving $>90 \%$ ee with proline as catalyst $\uparrow$
}

\begin{abstract}
Daria Sokolova (D) ${ }^{a}$ and Konrad Tiefenbacher (D) *ab
In previous work, we demonstrated that iminium-catalysed 1,4-reductions inside the supramolecular resorcinarene capsule display increased enantioselectivities as compared to their regular solution counterparts. Utilizing proline as the chiral catalyst, enantioselectivities remained below $80 \%$ ee. In this study, the reaction conditions were optimized by determining the optimal capsule loading and $\mathrm{HCl}$ content. Additionally, it was found that alcohol additives increase the enantioselectivity of the capsulecatalysed reaction. As a result, we report enantioselectivities of up to $92 \%$ ee for iminium-catalysed 1,4 reductions relying on proline as the sole chiral source. This is of high interest, as proline is unable to deliver high enantioselectivities for 1,4-reductions in a regular solution setting. Investigations into the role of the alcohol additive revealed a dual role: it not only slowed down the background reaction but also increased the capsule-catalysed reaction rate.
\end{abstract}

Received 4th June 2021

Accepted 5th July 2021

DOI: 10.1039/d1ra04333a

rsc.li/rsc-advances

Our understanding of capsule I-catalysis improved

\section{Introduction}

The self-assembled resorcinarene hexamer I (Fig. 1a), first reported by Atwood ${ }^{1}$ in the solid state in 1997, comprises one of the easiest to access molecular containers based on hydrogen bonds. It assembles from six resorcinarene units $\mathbf{1}$, readily accessible in one step on a large scale, and eight water molecules. ${ }^{1-3}$ Due to its dynamic nature, it can entrap guest molecules temporarily inside its cavity. ${ }^{2,4-9}$ The properties of $\mathbf{I}$ in solution were studied in detail, mainly by the groups of Rebek and Cohen. ${ }^{2-7,9-15}$ More recently, this container has started being explored as a container for other catalysts ${ }^{16}$ or as a catalyst itself. ${ }^{17,18}$ The field has been reviewed, ${ }^{19-23}$ and novel applications have been found very recently. ${ }^{24-27}$ In 2016, our group reported that iminium-catalysis can be performed inside capsule I (Fig. 1b). ${ }^{28,29}$ Later work by the Neri group extended iminium catalysis inside $\mathbf{I}$ to cycloadditions. ${ }^{30}$ The $\alpha, \beta$-unsaturated aldehyde first forms an iminium species with the chiral amine catalyst L-proline (3a). This iminium species is encapsulated due to the high affinity of the capsule $\mathbf{I}$ for cationic species. The subsequent reduction with Hantzsch ester 2, therefore, has to take place inside the confined environment of the capsule, delivering much higher enantioselectivities than in the absence of container $\mathbf{I}^{28,29}$

${ }^{a}$ Department of Chemistry, University of Basel, 4058 Basel, Switzerland. E-mail: konrad.tiefenbacher@unibas.ch

${ }^{b}$ Department of Biosystems Science and Engineering, ETH Zürich, 4058 Basel, Switzerland.E-mail: tkonrad@ethz.ch

$\dagger$ Electronic supplementary information (ESI) available: Experimental details, and copies of GC spectra. See DOI: 10.1039/d1ra04333a substantially over the last years. For instance, the importance of $\mathrm{HCl}$ as a co-catalyst for a selection of reactions inside $\mathbf{I}$ was elucidated. ${ }^{31}$ However, the influence of $\mathrm{HCl}$ on iminiumcatalysed reactions inside I remains unknown. This work aimed at (1) elucidating the role of $\mathrm{HCl}$ for the iminium catalysis inside $\mathbf{I}$; (2) reducing the amount of capsule catalyst required, as originally 26 mol\% were utilized; (3) optimizing the reaction conditions to improve the enantioselectivity. We
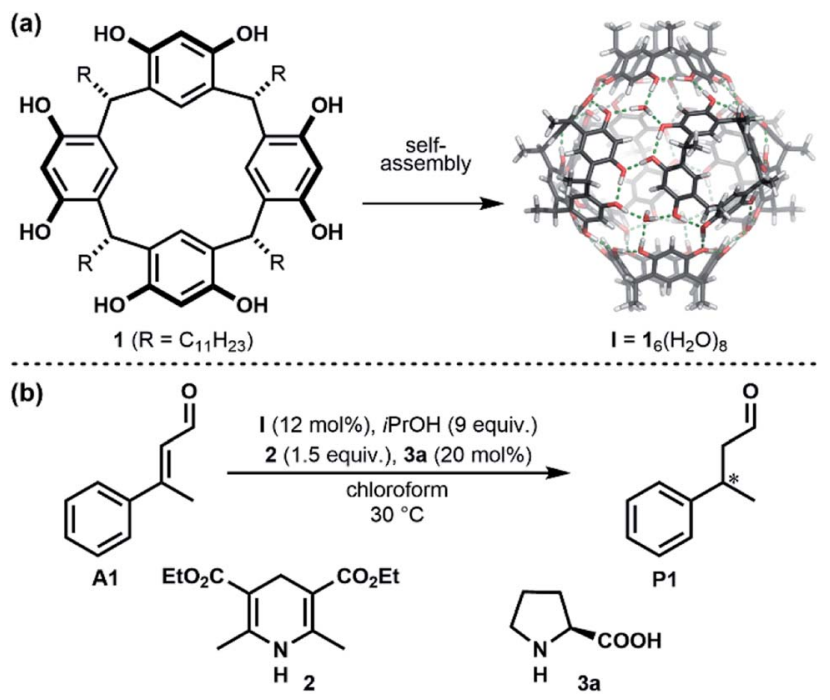

Fig. 1 (a) Self-assembly of monomer 1 into hexameric capsule I. (b) General scheme of iminium catalysed 1,4-reduction inside capsule I. 
here report the results of this effort. As a highlight of these studies, unprecedented enantioselectivities of up to $92 \%$ were achieved for the 1,4-reduction of $\alpha, \beta$-unsaturated aldehydes $^{32-34}$ inside capsule I using simple proline as the sole source of chiral information. This is of high interest as proline in a regular solution setting is unable to deliver high enantioselectivities for 1,4 -reductions. ${ }^{34}$

\section{Results and discussion}

As a first step, we aimed at reducing the unusually high capsule loading (26 mol\%). This loading was initially selected $^{28}$ to ensure complete uptake of proline (20 mol\%) and its iminium species. Utilizing the standard reaction conditions with substrate $\mathbf{A 1}$ (1 equiv. of $\mathbf{A 1}, c(\mathbf{A 1})=0.15 \mathrm{M}$ in chloroform, 1.5 equiv. of reducing agent $2,0.2$ equiv. of amine catalyst 3a; Section 3.2 in ESI $\dagger$ ), the influence of the capsule loading (8-26 mol\%) was explored (Fig. 2a). Interestingly, a peak selectivity ( $80 \%$ ee) was found at $12 \mathrm{~mol} \%$, which deteriorated both for increasing and decreasing loadings. This surprising finding may be a result of two opposing effects: (1) at low capsule loadings, the iminium reaction outside of the
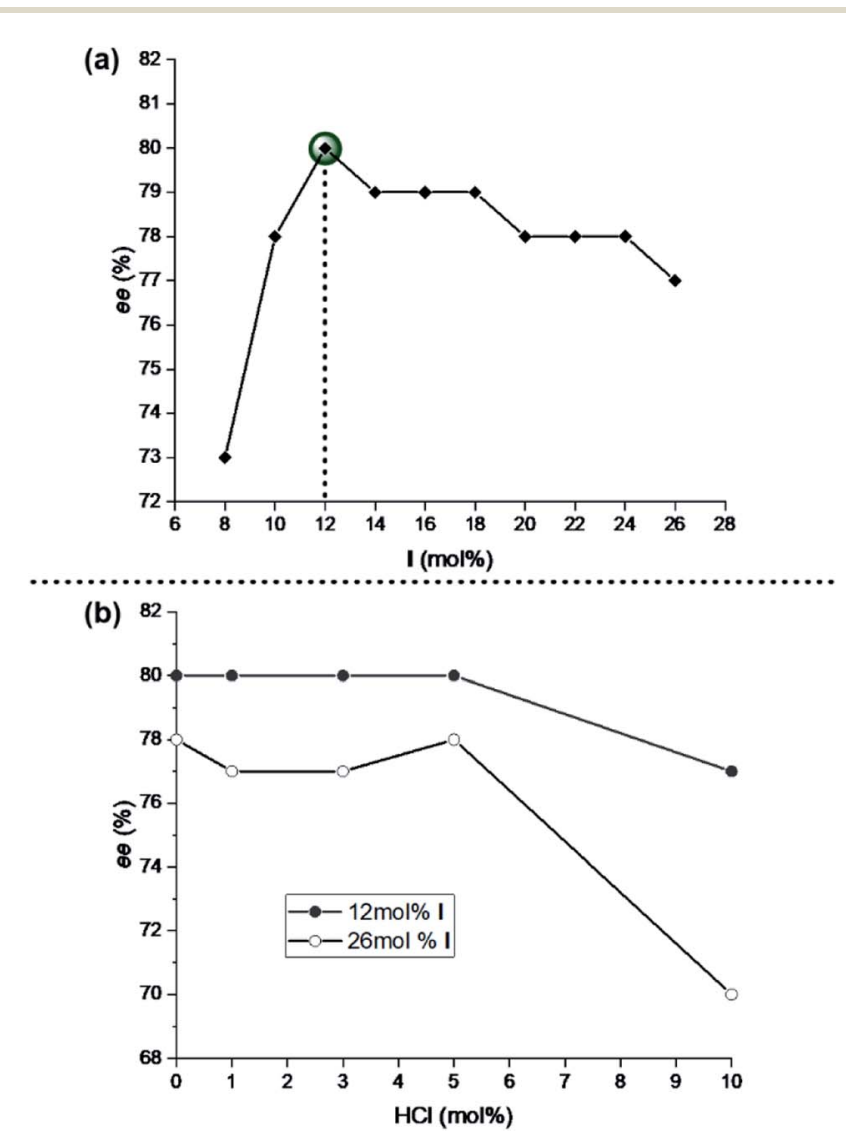

Fig. 2 (a) Optimization of the capsule I loading ( $8-26 \mathrm{~mol} \%$ ) under the standard reaction conditions using aldehyde $\mathrm{A} 1$ : 1 equiv. $\mathrm{A} 1, \mathrm{c}(\mathrm{A} 1)=$ $0.15 \mathrm{M}$ in chloroform, 1.5 equiv. 2, 0.2 equiv. $3 \mathrm{a}$. The optimal amount of I (12 mol\%) is highlighted. (b) Influence of the $\mathrm{HCl}$ content on the standard reaction utilizing either 12 or 26 mol\% of capsule I. Enantiomeric excesses were determined with chiral GC measurements. The values reported refer to measurements after $72 \mathrm{~h}$ of reaction time. capsule exerts a detrimental effect on the ee (background iminium reaction, $<12$ mol\%, entries 1 and 2 of Table S1 $\dagger$ ); (2) at higher capsule loadings, the racemic background reaction catalysed by capsule I itself starts dominating ( $>12 \mathrm{~mol} \%$, entries 4-10 of Table S1 $\dagger$ ). This background reaction is caused by the activation of the aldehyde substrate by the capsule itself (without proline) and leads to a racemic product as described in our previous work. ${ }^{28}$ Therefore, 12 mol\% I was selected for further studies.

In the previous work of our group, it was already demonstrated that Hantzsch ester 2 and L-proline (3a) are the most suitable hydride source and chiral catalyst, respectively. ${ }^{28,29}$ In this study, different L-proline loadings were evaluated with the newly optimized capsule I loading of $12 \mathrm{~mol} \%$. As can be seen in Table S2, $\uparrow$ the optimum was found at $20 \mathrm{~mol} \%$ of L-proline.

Next, the influence of $\mathrm{HCl}$ on the enantioselectivity of the standard reaction was explored. It was studied for two different capsule loadings: the optimized $12 \mathrm{~mol} \%$ and the previously utilized 26 mol\%. ${ }^{28,29}$ As can be seen in Fig. $2 \mathrm{~b}$, the enantioselectivity of the reaction does not benefit from $\mathrm{HCl}$ traces. Contrarily, larger $\mathrm{HCl}$ loadings ( $>5 \mathrm{~mol} \%$ ) even lead to a drop in enantioselectivity, presumably via an acid-catalysed racemic background reaction (Table S3†). A similar trend is observed for reactions with $26 \mathrm{~mol} \%$ of $\mathbf{I}$. Hence, the addition of $\mathrm{HCl}$ is not required for the iminium-catalysed 1,4-reductions inside capsule $\mathbf{I}$. This finding is in line with our previous report that not all reactions inside I depend on an acid co-catalyst. ${ }^{31}$

After having established the optimal capsule (12 mol\%) and proline (20 mol\%) loadings, and $\mathrm{HCl}$ content (none), further additives were investigated. Of particular interest were alcohol additives, as their interactions with the resorcinarene capsule are well documented..$^{15,35-37}$ The Cohen group investigated the incorporation of different alcohols into the resorcinarene capsule in solution and demonstrated that they can replace water molecules of the hydrogen bond network. ${ }^{15,36}$ Additionally, in the solid state it was reported that the incorporation of nPrOH into the hydrogen network of the resorcinarene hexamer results in $25 \%$ larger assemblies. ${ }^{38}$ These reports prompted us to investigate the influence of different alcohol additives on the iminium catalysis inside I. Indeed, initial

Table 1 Optimization of the alcohol additive under the standard reaction conditions: 1 equiv. $\mathrm{A} 1, \mathrm{C}(\mathrm{A} 1)=0.15 \mathrm{M}, 12 \mathrm{~mol} \%$ of $\mathrm{I}, 1.5$ equiv. 2, 0.2 equiv. $3 a^{a}$

\begin{tabular}{llccl}
\hline Entry & Alcohol & Yield (\%) & Conversion (\%) & ee (\%) \\
\hline 1 & - & $89 \pm 1$ & $93 \pm 1$ & $77 \pm 1(S)$ \\
2 & MeOH & $89 \pm 1$ & $93 \pm 1$ & $80 \pm 2(S)$ \\
3 & EtOH & $89 \pm 3$ & $94 \pm 2$ & $80 \pm 2(S)$ \\
4 & nPrOH & $90 \pm 1$ & $94 \pm 2$ & $80 \pm 2(S)$ \\
5 & iPrOH & $91 \pm 1$ & $94 \pm 2$ & $83 \pm 2(S)$ \\
6 & nBuOH & $89 \pm 2$ & $94 \pm 2$ & $80 \pm 2(S)$
\end{tabular}

${ }^{a}$ Conversions, yields, and enantiomeric excesses were determined with achiral and chiral GC measurements. The values reported refer to measurements after $72 \mathrm{~h}$ of reaction time. Reactions were performed in triplicate and standard deviations were determined 
tests revealed that alcohol additives increased the enantioselectivity of the standard reaction. An initial screening indicated that the best results were obtained with 9 equiv. of alcohol per capsule (Table $\mathrm{S} 4 \dagger$ ). Subsequently, several alcohol additives were screened (Table 1). As shown in Table 1 addition of each of the tested alcohols led to a slight increase of the ee, with $\mathrm{iPrOH}$ performing slightly better than the alternatives concerning yield and ee. Although the change in ee was not very big ( $6 \%$ ee, see entries 1 and 5 in Table 1), it was fully reproducible. The effect of the $\mathrm{iPrOH}$ additive was studied with a series of substrates (Table 2). In all cases, the combination of capsule I and $\mathrm{iPrOH}$ resulted in higher enantioselectivities. As reported in our previous work on the substrate scope, ${ }^{29}$ the effect of the capsule on substrate A5 was lower than in the other four cases; most likely due to the lack of an aromatic moiety that facilitates $\pi-\pi$ interactions with the capsule walls. Most interestingly, for two substrates (A2 and A3), enantioselectivities $>90 \%$ ee were achieved. This is remarkable, as proline is unable to deliver high enantioselectivities for 1,4reductions in a regular solution setting. ${ }^{34}$ Only in combination with the capsule catalyst and $\mathrm{PrOH}$, these high selectivities are observed.

How does iPrOH influence the enantioselectivity of the capsule-catalysed reaction? Initially, we investigated the influence of $\mathrm{iPrOH}$ on the capsule structure and size, as it was reported that alcohol incorporation can increase the size of the

Table 2 Results of reactions with different substrates under optimized conditions: 1 equiv. $\mathrm{A}, c(\mathrm{~A})=0.15 \mathrm{M}$ in chloroform, $12 \mathrm{~mol} \% \mathrm{I}, 1.5$ equiv. 2 , 0.2 equiv. 3a, 9 equiv. iPrOH, (bright-green). Comparison to reactions in the presence of $12 \mathrm{~mol} \%$ of capsule I, but the absence of iPrOH (darkgreen); reactions in the absence of capsule I, and the presence of 9 equiv. of iPrOH (dark-grey); reactions in the absence of both capsule I and iPrOH (light-grey) ${ }^{a}$

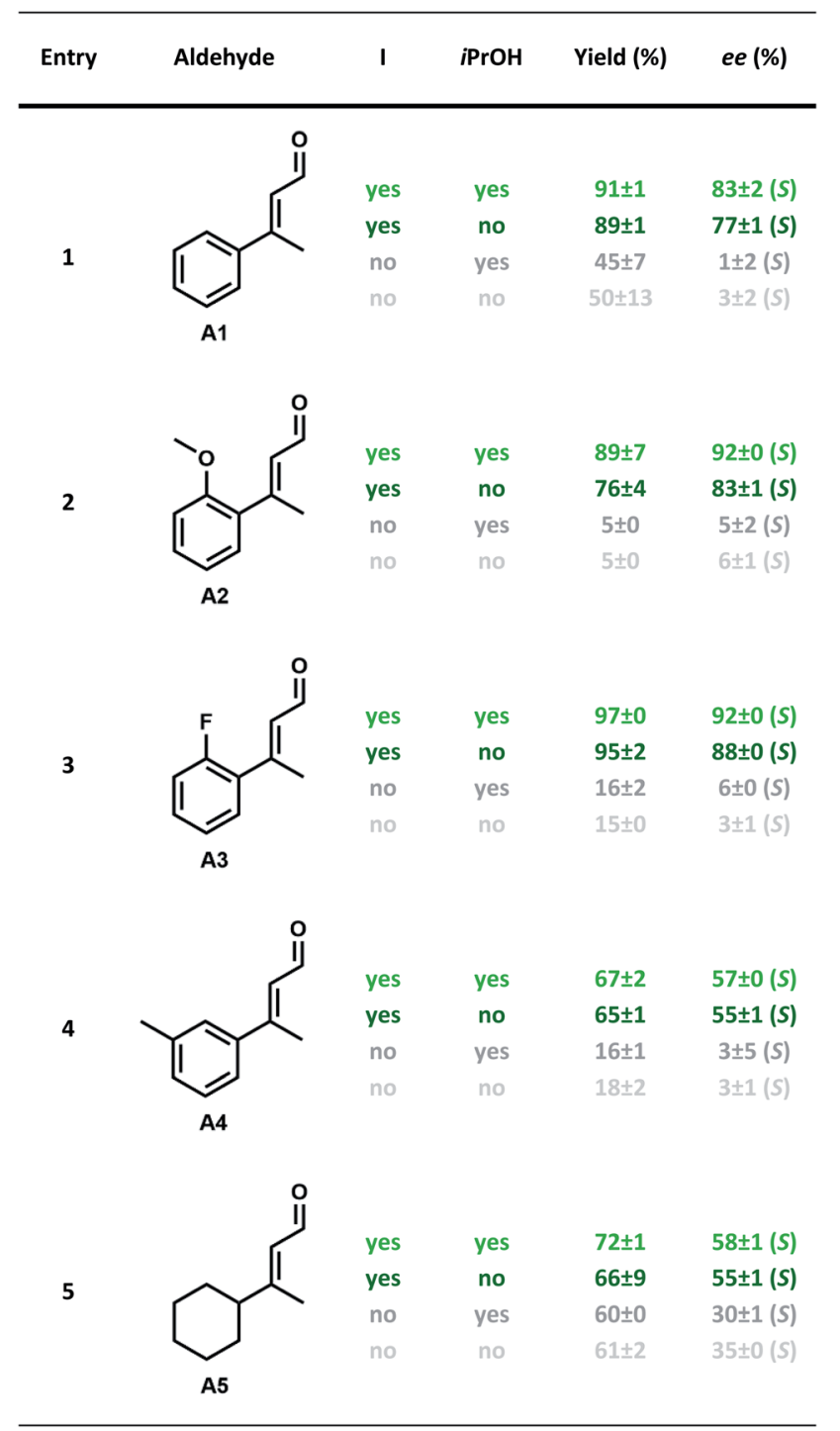

\footnotetext{
${ }^{a}$ Conversions, yields, and enantiomeric excesses were determined by GC measurements. The values reported refer to measurements after $72 \mathrm{~h}$ of reaction time. Reactions were performed in triplicate and standard deviations were determined
} 


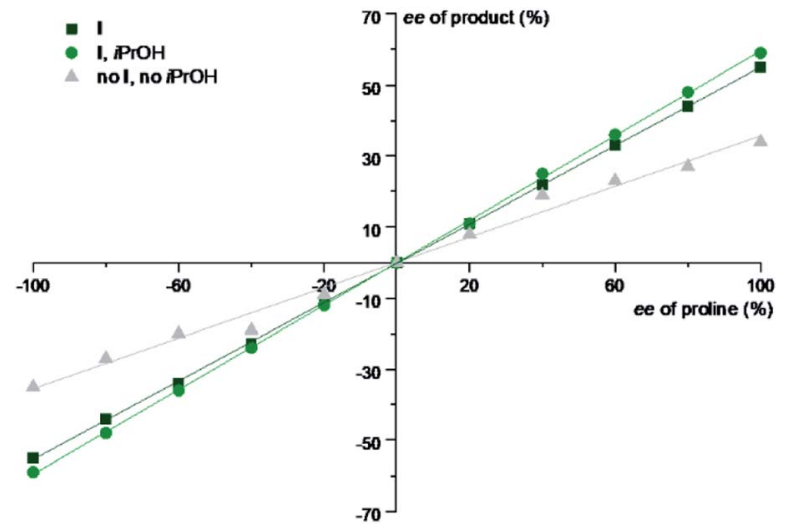

Fig. 3 Non-linear effects study of iminium catalysed 1,4-reduction under optimized conditions using aldehyde A5: 1 equiv. $\mathrm{A} 5, c(\mathrm{~A} 5)=$ $0.15 \mathrm{M}$ in chloroform, 1.5 equiv. $2,0.2$ equiv. of proline (mixtures of Land $\mathrm{D}$-proline of different ratios). Different reaction conditions (absence/presence of I and/or $\mathrm{iPrOH}$ ) were explored. Enantiomeric excesses were determined by chiral GC measurements. For more details, see Section 3.7 of the ESI. $\dagger$

capsule by $25 \%$, at least in the solid state. ${ }^{38}$ However, DOSYNMR data indicates that the size of the capsule does not change upon the addition of $\mathrm{iPrOH}$ (see Section 5 of the ESI $\dagger$ for diffusion coefficients). This is in line with observations in solution from the Cohen group, who reported the replacement of water molecules in the hydrogen bond network by iPrOH without a significant change in the assembly size. ${ }^{36}$

Another hypothesis centered on the idea that the alcohol additive might influence proline-proline interactions in the capsule.

If such interactions play a role, non-linear effects (NLE) should be observable. Therefore, a NLE study was performed. Substrate A5 was chosen since it yields the highest ee in the absence of the capsule (Table 2) and thus facilitates the observation of changes. In all the cases, linear graphs were obtained (Fig. 3). Accordingly, we can exclude iPrOH-effected changes in the proline-proline interactions as the source of the increased enantioselectivity.

Subsequently, we decided to investigate the initial rates and initial ee of the reaction, in order to elucidate the role of the iPrOH additive. Substrate A2 was chosen for this study as it displays the fastest kinetics. Four reactions (presence/absence of capsule I; presence/absence of alcohol additive) were studied in parallel. The capsule-free reactions turned out to be faster than the capsule-mediated ones, although they plateaued at approx. 40$50 \%$ conversion (Fig. 4a). Slowed down kinetics for iminium catalysis inside the capsule is to be expected as the Hantzsch ester and the iminium species have to be co-encapsulated for conversion. More interestingly, the alcohol additive suppressed the reaction rate in the absence of capsule; however, it increased the rate in the presence of capsule I. A different trend was observed when following the initial enantioselectivity of the reaction (Fig. 4b). While the influence of alcohol additive was negligible for the reactions without capsule, the capsule-mediated reaction benefited from the additive ( $92 \%$ ee $v$ s. $84 \%$ ee).

We interpret the results the following way: the increased enantioselectivity in the presence of alcohol additive and
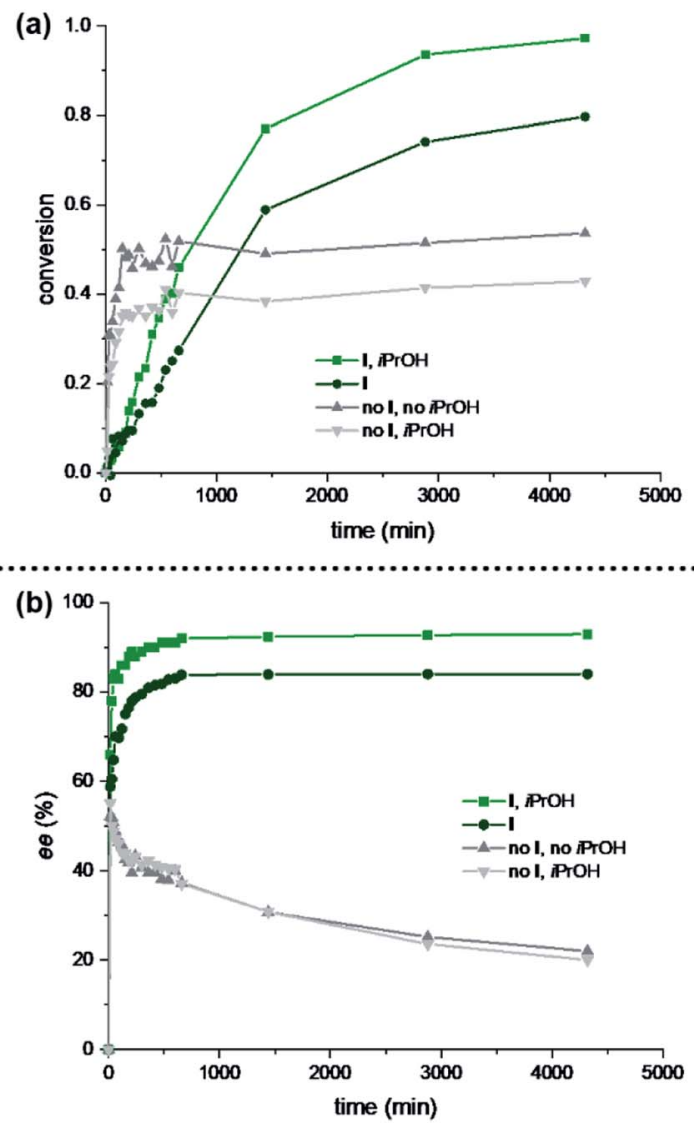

Fig. 4 Influence of $\mathrm{iPrOH}$ on the initial rates and enantiomeric excesses of the reaction under optimized conditions using aldehyde A2: 1 equiv. $A 2, c(A 2)=0.15 \mathrm{M}$ in chloroform, 1.5 equiv. $2,0.2$ equiv. $3 a$. Conversions and ees were determined with achiral and chiral GC measurements, respectively. (a) Conversions of reactions in the presence/absence of capsule I (12 mol\% if present); the presence/ absence of alcohol additive (9 equiv. if present). (b) Enantiomeric excesses of reactions in the presence/absence of capsule I (12 mol\% if present); the presence/absence of alcohol additive ( 9 equiv. if present). For more details, see Section 3.8 of the ESI. $\dagger$

capsule is likely a consequence of two effects. (1) The reduced background reaction of the free iminium species (compare grey lines in Fig. 4a) that yields low ees. (2) The increased reaction rate in the capsule (compare green lines in Fig. 4a). The acceleration of the reaction in the presence of alcohol additive and capsule (light-green line in Fig. 4a) likely stems from faster exchange kinetics of the reagents in/out of the capsule, as it is known that polar additives destabilize the hydrogen-bonding network. $^{2}$ These results indicate that the proline-catalysed reaction inside capsule $\mathbf{I}$ is highly enantioselective, and is only reduced to some extent by the background reaction outside of the capsule that delivers basically racemic product.

\section{Conclusions}

We present optimized reaction conditions for the iminiumcatalysed 1,4-reduction of $\alpha, \beta$-unsaturated aldehyde inside the supramolecular capsule I. The capsule loading was successfully 
reduced from 26 to $12 \mathrm{~mol} \%$. Furthermore, it was established that $\mathrm{HCl}$ is not required as a co-catalyst. Most interestingly, it was found that alcohol additives have a beneficial role concerning the enantioselectivities observed. In two cases, products with $92 \%$ ee were formed. To our knowledge, this is the first time that such high enantioselectivities were observed for iminium-catalysed 1,4-reductions utilizing proline as the sole chiral source. While proline performs poorly in solution, the increased interactions inside the confined space of I lead to a dramatic increase in enantioselectivity. According to our initial hypothesis, ${ }^{28,29}$ this ee-increase stems from a selective shielding of one side of the iminium species by the inner wall of capsule I. This study demonstrates that this enantioselectivity can be further increased by alcohol additives that not only decrease the background reaction but also accelerate the capsule-catalysed process. We are convinced that these results not only strengthen our understanding of confinement catalysis but will also be transferable to other reaction classes.

\section{Author contributions}

K. T. conceived the original idea and supervised the project. D. S. carried out and analysed the experiments. D. S. and K. T. compiled the manuscript.

\section{Conflicts of interest}

There are no conflicts to declare.

\section{Acknowledgements}

This work was supported by funding from the European Research Council Horizon 2020 Programme [ERC Starting Grant 714620-TERPENECAT]. We also thank Suren Nemat for helpful discussions.

\section{Notes and references}

1 L. R. MacGillivray and J. L. Atwood, A chiral spherical molecular assembly held together by 60 hydrogen bonds, Nature, 1997, 389, 469-472.

2 L. Avram and Y. Cohen, Spontaneous formation of hexameric resorcinarene capsule in chloroform solution as detected by diffusion NMR, J. Am. Chem. Soc., 2002, 124, 15148-15149.

3 L. Avram, Y. Cohen and J. Rebek, Recent advances in hydrogen-bonded hexameric encapsulation complexes, Chem. Commun., 2011, 47, 5368-5375.

4 A. Shivanyuk and J. Rebek, Reversible encapsulation by selfassembling resorcinarene subunits, Proc. Natl. Acad. Sci. U. S. A., 2001, 98, 7662-7665.

5 L. Avram and Y. Cohen, Discrimination of guests encapsulation in large hexameric molecular capsules in solution: pyrogallol[4]arene versus resorcin[4]arene capsules, J. Am. Chem. Soc., 2003, 125, 16180-16181.
6 L. Avram and Y. Cohen, Effect of a cationic guest on the characteristics of the molecular capsule of resorcinarene: a diffusion NMR study, Org. Lett., 2003, 5, 1099-1102.

7 M. Yamanaka, A. Shivanyuk and J. Rebek, Kinetics and thermodynamics of hexameric capsule formation, J. Am. Chem. Soc., 2004, 126, 2939-2943.

8 L. Avram and Y. Cohen, Self-recognition, structure, stability, and guest affinity of pyrogallol[4]arene and resorcin[4]arene capsules in solution, J. Am. Chem. Soc., 2004, 126, 1155611563.

9 L. Avram and Y. Cohen, Self-assembly of resorcin[4]arene in the presence of small alkylammonium guests in solution, Org. Lett., 2008, 10, 1505-1508.

10 A. Shivanyuk and J. Rebek, Assembly of resorcinarene capsules in wet solvents, J. Am. Chem. Soc., 2003, 125, 3432-3433.

11 T. Evan-Salem, I. Baruch, L. Avram, Y. Cohen, L. C. Palmer and J. Rebek, Resorcinarenes are hexameric capsules in solution, Proc. Natl. Acad. Sci. U. S. A., 2006, 103, 1229612300.

12 E. S. Barrett, T. J. Dale and J. Rebek, Assembly and exchange of resorcinarene capsules monitored by fluorescence resonance energy transfer, J. Am. Chem. Soc., 2007, 129, 3818-3819.

13 E. S. Barrett, T. J. Dale and J. Rebek, Stability, dynamics, and selectivity in the assembly of hydrogen-bonded hexameric capsules, J. Am. Chem. Soc., 2008, 130, 2344-2350.

14 S. Slovak and Y. Cohen, In-out interactions of different guests with the hexameric capsule of resorcin[4]arene, Supramol. Chem., 2010, 22, 803-807.

15 S. Slovak, L. Avram and Y. Cohen, Encapsulated or not encapsulated? mapping alcohol sites in hexameric capsules of resorcin[4] arenes in solution by diffusion NMR spectroscopy, Angew. Chem., Int. Ed., 2010, 49, 428-431.

16 A. Cavarzan, A. Scarso, P. Sgarbossa, G. Strukul and J. N. H. Reek, Supramolecular control on chemo- and regioselectivity via encapsulation of (NHC)-Au catalyst within a hexameric self-assembled host, J. Am. Chem. Soc., 2011, 133, 2848-2851.

17 G. Bianchini, G. La Sorella, N. Canever, A. Scarso and G. Strukul, Efficient isonitrile hydration through encapsulation within a hexameric self-assembled capsule and selective inhibition by a photo-controllable competitive guest, Chem. Commun., 2013, 49, 5322-5324.

18 Q. Zhang and K. Tiefenbacher, Hexameric resorcinarene capsule is a Brønsted acid: investigation and application to synthesis and catalysis, J. Am. Chem. Soc., 2013, 135, 16213-16219.

19 Q. Zhang, L. Catti, L. D. Syntrivanis and K. Tiefenbacher, En route to terpene natural products utilizing supramolecular cyclase mimetics, Nat. Prod. Rep., 2019, 36, 1619-1627.

20 S. Gambaro, M. De Rosa, A. Soriente, C. Talotta, G. Floresta, A. Rescifina, C. Gaeta and P. Neri, A hexameric resorcinarene capsule as a hydrogen bonding catalyst in the conjugate addition of pyrroles and indoles to nitroalkenes, $\mathrm{Org}$. Chem. Front., 2019, 6, 2339-2347. 
21 Y. Zhu, J. Rebek and Y. Yu, Cyclizations catalyzed inside a hexameric resorcinarene capsule, Chem. Commun., 2019, 55, 3573-3577.

22 I. Némethová, L. D. Syntrivanis and K. Tiefenbacher, Molecular capsule catalysis: ready to address current challenges in synthetic organic chemistry?, Chimia, 2020, 74, 561-568.

23 C. Gaeta, P. La Manna, M. De Rosa, A. Soriente, C. Talotta and P. Neri, Supramolecular catalysis with self-assembled capsules and cages: what happens in confined spaces, ChemCatChem, 2021, 13, 1638-1658.

24 S. Merget, L. Catti, G. Piccini and K. Tiefenbacher, Requirements for terpene cyclizations inside the supramolecular resorcinarene capsule: bound water and its protonation determine the catalytic activity, J. Am. Chem. Soc., 2020, 142(9), 4400-4410.

25 L. D. Syntrivanis, I. Némethová, D. Schmid, S. Levi, A. Prescimone, F. Bissegger, D. T. Major and K. Tiefenbacher, Four-step access to the sesquiterpene natural product presilphiperfolan-1 $\beta$-ol and unnatural derivatives via supramolecular catalysis, J. Am. Chem. Soc., 2020, 142, 5894-5900.

26 P. La Manna, C. Talotta, M. De Rosa, A. Soriente, C. Gaeta and P. Neri, An atom-economical method for the formation of amidopyrroles exploiting the self-assembled resorcinarene capsule, Org. Lett., 2020, 22, 2590-2594.

27 S. Gambaro, C. Talotta, P. Della Sala, A. Soriente, M. De Rosa, C. Gaeta and P. Neri, Kinetic and thermodynamic modulation of dynamic imine libraries driven by the hexameric resorcinarene capsule, J. Am. Chem. Soc., 2020, 142, 14914-14923.

28 T. M. Bräuer, Q. Zhang and K. Tiefenbacher, Iminium catalysis inside a self-assembled supramolecular capsule: modulation of enantiomeric excess, Angew. Chem., Int. Ed., 2016, 55, 7698-7701.

29 T. M. Bräuer, Q. Zhang and K. Tiefenbacher, Iminium catalysis inside a self-assembled supramolecular capsule: scope and mechanistic studies, J. Am. Chem. Soc., 2017, 139, 17500-17507.

30 P. La Manna, M. De Rosa, C. Talotta, C. Gaeta, A. Soriente, G. Floresta, A. Rescifina and P. Neri, The hexameric resorcinarene capsule as an artificial enzyme: Ruling the regio and stereochemistry of a 1,3-dipolar cycloaddition between nitrones and unsaturated aldehydes, Org. Chem. Front., 2018, 5, 827-837.

$31 \mathrm{~J}$. M. Köster and K. Tiefenbacher, Elucidating the importance of hydrochloric acid as a cocatalyst for resorcinarene-capsule-catalyzed reactions, ChemCatChem, 2018, 10, 2941-2944.

32 W. Y. Jung, M. T. Hechavarria Fonseca, N. Vignola and B. List, Metal-free, organocatalytic asymmetric transfer hydrogenation of $\alpha, \beta$-unsaturated aldehydes, Angew. Chem., Int. Ed., 2004, 44, 108-110.

33 J. W. Yang, M. T. Hechavarria Fonseca and B. List, A metalfree transfer hydrogenation: organocatalytic conjugate reduction of $\alpha, \beta$-unsaturated aldehydes, Angew. Chem., Int. Ed., 2004, 43, 6660-6662.

34 S. G. Ouellet, J. B. Tuttle and D. W. C. MacMillan, Enantioselective organocatalytic hydride reduction, J. Am. Chem. Soc., 2005, 127, 32-33.

35 B. Schnatwinkel, I. Stoll, A. Mix, M. V. Rekharsky, V. V. Borovkov, Y. Inoue and J. Mattay, Monomeric, dimeric and hexameric resorcin[4]arene assemblies with alcohols in apolar solventswzy, Chem. Commun., 2008, 3873-3875.

36 S. Slovak and Y. Cohen, The effect of alcohol structures on the interaction mode with the hexameric capsule of resorcin[4]arene, Chem.-Eur. J., 2012, 18, 8515-8520.

37 O. Ugono and K. T. Holman, An achiral form of the hexameric resorcin[4] arene capsule sustained by hydrogen bonding with alcohols, Chem. Commun., 2006, 2144-2146.

38 R. M. Payne and C. L. Oliver, A propanol-seamed: Cmethylcalix[4]resorcinarene hexamer accessible via solution crystallization, liquid-assisted grinding and vapour sorption, CrystEngComm, 2018, 20, 1919-1922. 